\title{
Warfarin Hypersensitivity Due to Gluten-Sensitive Enteropathy: A Case Study
}

\author{
SARA KWOLEK, PAULA DEMING
}

\begin{abstract}
:
A 53 year old female who was maintained on long-term warfarin therapy due to history of pulmonary embolism, repeatedly presents with an abnormally prolonged Prothrombin Time (PT) and Activated Partial Thromboplastin Time (APTT). After many asymptomatic episodes were corrected with Vitamin K therapy to temporarily reverse the effects of the warfarin, the cause of the apparent coagulopathy was further investigated. Factor Activity Assays of the common pathway factors II, IX, and X all revealed critically low values; below the threshold even a loading dose of warfarin is typically capable of eliciting. The patient tested strongly positive for Tissue Transglutaminase IgA, which is highly suggestive of a gluten-sensitive enteropathy. One effect of this condition is malabsorption due to flattened intestinal villi. ${ }^{1}$ The patient was determined to have an acquired vitamin $\mathrm{K}$ deficiency secondary to gluten-sensitive enteropathy. Her condition was exacerbated by the long-term warfarin therapy, resulting in the prolonged PT and PTT. The patient was treated with vitamin $\mathrm{K}$ therapy, which reversed the deficiency and corrected her abnormal coagulation results.
\end{abstract}

INDEX TERMS: Warfarin, International normalized ratio, Prothrombin time, Gluten sensitive enteropathy, Vitamin K deficiency.

ABBREVIATIONS: PT- prothrombin time, APTTactivated partial thromboplastin time, INRinternational normalized ratio, $\mathrm{CD}$ - celiac disease

Clin Lab Sci 2012;25(2):78

Sara Kwolek, BS MLS(ASCP) ${ }^{C M}$, Laboratory Services, Glens Falls Hospital, Glens Falls, NY

Paula Deming, Ph.D., MLS(ASCP) ${ }^{C M}$, Medical Laboratory and Radiation Sciences, University of Vermont Burlington, VT
Address for Correspondence: Paula Deming, Ph.D., MLS(ASCP) ${ }^{C M}$, Medical Laboratory and Radiation Sciences, University of Vermont, 106 Carrigan Drive, Rm. 302 Rowell Building, Burlington, VT 05405, (802) 6562506, paula.deming@uvm.edu

In February 2010, a 53-year old female patient with a history of pulmonary embolism presented for a routine prothrombin time (PT) and international normalized ratio (INR) to monitor long-term warfarin therapy as a result of a pulmonary embolism. The initial PT result was prolonged at 64 seconds (normal reference range is 11.1-14.7 seconds, Table 1) and the INR was 7.0, which is well outside the therapeutic range of 2.0-3.5. ${ }^{2}$ Over the next week, the INR remained excessively prolonged, but she was asymptomatic. Vitamin K therapy normalized the patient's INR within the therapeutic range. In June 2010, she presented with a second asymptomatic INR prolongation, over a two day period (Table 1). At this time, the activated partial thromboplastin time (APTT) was also markedly prolonged but it was corrected when mixed 1:1 with normal control plasma (Table 1), even following a one hour incubation at $37^{\circ} \mathrm{C}$ (Table 1). These results suggested a clotting factor deficiency and ruled out the presence of a weak or time-dependent inhibitor such as a factor VIII inhibitor or a lupus anticoagulant. The patient was again given vitamin $\mathrm{K}$, which normalized the INR to 1.4 .

Six months later, the patient was admitted to the hospital with a gastrointestinal bleed, hematuria and an abnormally prolonged PT with an INR of 9.4. Two units of fresh frozen plasma were transfused, and her INR normalized within the therapeutic range. In March 2011, she presented with excessively prolonged coagulation results despite being asymptomatic (Table 1). She was admitted to the hospital for closer monitoring with a diagnosis of 'elevated risk of bleed'. PT and APTT mixing studies showed a correction when patient plasma was mixed 1:1 with normal 
control plasma (Table 1), which is consistent with a factor deficiency. In light of the patient's low hemoglobin and hematocrit $(8.5 \mathrm{~g} / \mathrm{dl}$ and $25.5 \%$, respectively); she was transfused with two units typespecific packed red blood cells, and two units of fresh frozen plasma. This increased the hemoglobin to 12 $\mathrm{g} / \mathrm{dl}$ and hematocrit to $36.2 \%$ and normalized the patient's PT time over the next 24 hours. The APTT time also dropped significantly by the next day but it was still elevated at 61 seconds.

Table 1. Laboratory Data

\begin{tabular}{|c|c|c|c|}
\hline Variable & $\begin{array}{l}\text { Reference Ranges } \\
\text { (units) }\end{array}$ & $\begin{array}{c}\text { Patient } \\
\text { result } \\
\text { June, } 2010\end{array}$ & $\begin{array}{c}\text { Patient } \\
\text { result } \\
\text { March, 2011 }\end{array}$ \\
\hline $\mathrm{PT}^{*}$ & $11.1-14.7 \mathrm{sec}$ & $\begin{array}{l}\text { Day 1: } 80.7 \\
\text { Day 2: } 61\end{array}$ & 93.3 \\
\hline $\mathrm{APTT}^{*}$ & $20.9-35.3 \mathrm{sec}$ & $\begin{array}{l}\text { Day 1: } 94.9 \\
\text { Day 2: } 110.1\end{array}$ & 138.6 \\
\hline Therapeutic INR ${ }^{[2]}$ & $2-3.5$ & $\begin{array}{l}\text { Day 1: } 9.4 \\
\text { Day 2: } 6.6\end{array}$ & 13 \\
\hline 1:1 mix* & $20.9-35.3 \mathrm{sec}$ & Day 2: 33.5 & 32.5 \\
\hline $\begin{array}{l}\text { APTT } \\
1 \text { hr } @ 37^{\circ} \mathrm{C}^{*}\end{array}$ & $20.9-35.3 \mathrm{sec}$ & 104 & 118.2 \\
\hline $\begin{array}{l}\text { APTT } 1: 1 \mathrm{mix}, \\
1 \mathrm{hr} @ 37^{\circ} \mathrm{C}^{*}\end{array}$ & $20.9-35.3 \mathrm{sec}$ & 34.9 & 32.3 \\
\hline PT $1: 1 \mathrm{mix}^{*}$ & $11.1-14.7 \mathrm{sec}$ & & 14.9 \\
\hline dRVVT $[3]$ & $33-44 \mathrm{sec}$ & & 91 \\
\hline dRVVT $1: 1 \operatorname{mix}^{[3]}$ & $33-44 \mathrm{sec}$ & & 39 \\
\hline Factor II activity ${ }^{[14]}$ & $50-150 \%$ & & $<5$ \\
\hline Factor VIII activity ${ }^{[14]}$ & $50-150 \%$ & & 138 \\
\hline Factor IX activity ${ }^{[14]}$ & $50-150 \%$ & & 3 \\
\hline Factor X activity ${ }^{[14]}$ & $50-150 \%$ & & $<7$ \\
\hline
\end{tabular}

* Reference ranges were calculated at Glens Falls Hospital from a group of healthy adults. Reference ranges are affected by many variables, including the patient population and laboratory methods used and therefore may vary from institution to institution.

The patient's results to this point were consistent with a deficiency in one of the common pathway factors and therefore to investigate this further, a Dilute Russell's Viper Venom Time (dRVVT) was ordered. Russell Viper venom activates factor $\mathrm{X}$ and factor $\mathrm{V}$, thus initiating the clotting cascade through direct activation of the common pathway. The test is very sensitive to inhibitors of the prothrombinase complex and a dRVVT mixing study (patient plasma 1:1 normal plasma) may be used to differentiate between deficiencies of factors $\mathrm{X}, \mathrm{V}$, II or fibrinogen and the presence of inhibitors. ${ }^{3}$ The patient's initial dRVVT time was prolonged but was reversed to within the normal range upon mixing 1:1 with normal control plasma (Table 1). Although these results are indicative of a common pathway factor deficiency, the results of individual factor activity assays ruled out this possibility as critically low levels were found for the common pathway factors II and X, and also for factor IX, an intrinsic pathway factor (Table 1). Given that factors II, $\mathrm{X}$, IX (all found to be critically low in this patient) and VII (the activity of which was not tested in this patient) are dependent upon vitamin $\mathrm{K}$ for functional activation in the liver, a deficiency in vitamin $\mathrm{K}$ was suspected

Vitamin $\mathrm{K}$ is absorbed from the diet in the small intestines and is required in its reduced form as a cofactor for $\gamma$-glutamyl-carboxylase to convert inactive factors II, VII, IX, and X to the functionally carboxylated active forms necessary for coagulation. Warfarin exerts its anticoagulant effects by inhibiting hepatic vitamin $\mathrm{K}$ epoxide reductase (VKOR), which prevents the recycling and activation of vitamin $\mathrm{K}$ and thus decreases the amount of functional clotting factors. ${ }^{4}$ Although vitamin K-dependent factors are notably affected by warfarin, their activity is not typically compromised to the degree observed in this patient. ${ }^{2}$ A study of the effects of $10 \mathrm{mg}$ loading doses of warfarin conducted by Harrison et al showed that 36 hours post-dose, the factor activity levels were well above the patient described in this case. ${ }^{5} \mathrm{~A}$ loading dose is a larger dose given to a patient just beginning therapy; this dose is gradually lessened until a dose is found that will maintain a therapeutic INR level. The average factor II activity level in the patients receiving a loading dose was reported to be $74 \%$, which is considerably higher than the $<5 \%$ observed for our patient on maintenance therapy. Moreover, while only about one third of patients who received a loading dose of warfarin had INR values of greater than 3.0 at 60 hours post dose, ${ }^{5}$ the patient described here had INR values upwards to 13 despite being asymptomatic.

One last test in the investigation into this patient's apparent coagulopathy was a Tissue Transglutaminase IgA Antibody screen, or tTG IgA. The tTG IgA test utilizes the enzyme-linked immunosorbent assay (ELISA) method, and is both very sensitive and very specific for antibodies to tissue transglutaminase, a pathological marker of gluten-sensitive enteropathy. ${ }^{1}$ tTG is a ubiquitous calcium-dependent enzyme important in crosslinking extracellular matrix proteins 
to stabilize damaged tissue. It appears to play an important role in gluten-sensitive enteropathies such as celiac disease by forming a cross-linked complex with the wheat protein, gliadin. ${ }^{1}$ The tTG-gliadin complex stimulates a $\mathrm{T}$ cell-mediated immune response with an autoimmune component in susceptible individuals. This immune response causes direct damage to the intestinal villi causing them to become flattened. Depending on the severity of the disease there can be a range of intestinal malabsorption symptoms such as diarrhea, vomiting, abdominal pain, bloating, as well as extra-intestinal symptoms including iron-deficiency anemia, unexplained folic acid deficiency and a hemorrhagic syndrome caused by vitamin K deficiency. ${ }^{1}$ The patient's tTG IgA result was strongly positive, which indicated that she had an underlying glutensensitive enteropathy. ${ }^{6}$

When all of these test results were considered, it was determined that this patient's excessively prolonged PT/INR and APTT results were due to an acquired vitamin $\mathrm{K}$ deficiency secondary to gluten-sensitive enteropathy. The warfarin regimen this patient was maintained on following a pulmonary embolism exacerbated the deficiency, causing an increased sensitivity to the anticoagulant, hence the excessively prolonged coagulation test results. Mild vitamin $\mathrm{K}$ deficiency is reported to be common in patients with chronic gastrointestinal disorders ${ }^{7}$ and although bleeding due to malabsorption of vitamin $\mathrm{K}$ in patients with celiac disease occurs rarely, it has been reported in the literature since the 1970 's. ${ }^{8}$ A study conducted by Cavallaro et al., found that $18.5 \%$ of untreated celiac patients had a prolonged PT. ${ }^{9}$ Moreover, the prolonged PT was significantly related to all of the markers of severe malabsorption including anemia and abnormal iron proteins. ${ }^{?}$

It is not uncommon for patients taking warfarin to present asymptomatically with excessive prolongation of their INR values. ${ }^{10}$ It has been reported that more than $11 \%$ of patients receiving warfarin for one year will have an INR value greater than the target range. ${ }^{11}$ Changes in the dose increment of warfarin, fluxes in dietary vitamin $\mathrm{K}$ intake, polymorphisms in the genes that encode the hepatic CYP2CP enzyme which metabolizes warfarin and vitamin $\mathrm{K}$ epoxide reductase, as well as the addition of unrelated medications that interact with warfarin, may contribute to asymptomatic increases in PT/INR in patients on long-term warfarin therapy. ${ }^{12}$ It is entirely possible that one or more of these factors contributed to the asymptomatic fluctuations in PT/INR observed in this patient, which were further aggravated by her underlying vitamin $\mathrm{K}$ deficiency. Low-dose therapy with vitamin $\mathrm{K}$ is effective in controlling asymptomatic PT prolongation ${ }^{10,13}$ and was used successfully with this patient to bring the INR back into the therapeutic range and correct her underlying deficiency.

\section{REFERENCES}

1. Volta, U. and V. Villanacci, Celiac disease: diagnostic criteria in progress. Cell Mol Immunol, 2011;8(2):96-102.

2. Gulati, G., et al., International normalized ratio versus plasma levels of coagulation factors in patients on vitamin $\mathrm{K}$ antagonist therapy. Arch Pathol Lab Med, 2011;135(4):490-4.

3. Dilute Russell Viper Venom Time (dRVVT) with Reflex to dRVVT 1:1 Mix \& Confirmation [Internet] ARUP Laboratories: A National Reference Laboratory [cited 2011 October 24, 2011]; Available from: http://www.aruplab.com/ guides/ug/ tests/0030461.jsp.

4. Choonara, I.A., et al., The relationship between inhibition of vitamin $\mathrm{K} 1$ 2,3-epoxide reductase and reduction of clotting factor activity with warfarin. $\mathrm{Br} \mathrm{J}$ Clin Pharmacol, 1988;25(1):1-7.

5. Harrison, L., et al., Comparison of 5-mg and 10-mg loading doses in initiation of warfarin therapy. Ann Intern Med, 1997;126(2):133-6.

6. Tissue Transglutaminase Antibody (tTG) IgA: ARUP Lab Tests. [Internet] ARUP Laboratories: A National Reference Laboratory [cited 2011 October 17, 2011]; Available from: http://www.aruplab.com/guides/ug/tests/0097709.jsp.

7. Krasinski, S.D., et al., The prevalence of vitamin K deficiency in chronic gastrointestinal disorders. Am J Clin Nutr, 1985;41(3):639-43.

8. Chen, C.S., E.U. Cumbler, and A.T. Triebling, Coagulopathy due to celiac disease presenting as intramuscular hemorrhage. J Gen Intern Med, 2007;22(11):1608-12.

9. Cavallaro, R., et al., Prevalence and clinical associations of prolonged prothrombin time in adult untreated coeliac disease. Eur J Gastroenterol Hepatol, 2004;16(2):219-23.

10. Crowther, M.A. and S. Wilson, Vitamin K for the treatment of asymptomatic coagulopathy associated with oral anticoagulant therapy. J Thromb Thrombolysis, 2003;16(1-2):69-72.

11. Turpie, A.G., et al., A comparison of aspirin with placebo in patients treated with warfarin after heart-valve replacement. N Engl J Med, 1993;329(8):524-9.

12. Habib, G., et al., Excessive prolongation of prothrombin time among patients treated with warfarin and admitted to the emergency room. Eur J Intern Med, 2008;19(2):129-34.

13. Crowther, M.A., et al., Low-dose oral vitamin $\mathrm{K}$ reliably reverses over-anticoagulation due to warfarin. Thromb Haemost, 1998;79(6):1116-8.

14. Harmening, D.M., Clinical Hematology and Fundamentals of Hemostasis. 5th ed. 2009, Philadelphia: F.A. Davis. 